\title{
THE RADON-NIKODYM THEOREM FOR THE BOCHNER INTEGRAL
}

\author{
BY \\ M. A. RIEFFEL $\left({ }^{1}\right)$
}

Our Main Theorem, which we believe to be the first general Radon-Nikodym theorem for the Bochner integral, is

Main Theorem. Let $(X, S, \mu)$ be a $\sigma$-finite positive measure space and let $B$ be a Banach space. Let $m$ be a B-valued measure on $S$. Then $m$ is the indefinite integral with respect to $\mu$ of a B-valued Bochner integrable function on $X$ if and only if

(1) $m$ is $\mu$-continuous, that is, $m(E)=0$ whenever $\mu(E)=0, E \in S$,

(2) the total variation, $|m|$, of $m$ is a finite measure,

(3) locally $m$ somewhere has compact average range, that is, given $E \in S$ with $0<\mu(E)<\infty$ there is an $F \subseteq E$ such that $\mu(F)>0$ and

$$
A_{F}(m)=\left\{m\left(F^{\prime}\right) / \mu\left(F^{\prime}\right): F^{\prime} \subseteq F, \mu\left(F^{\prime}\right)>0\right\}
$$

is relatively (norm) compact, or equivalently

(3') locally $m$ somewhere has compact direction, that is, given $E \in S$ with $0<\mu(E)<\infty$ there is an $F \subseteq E$ and a compact subset, $K$, of $B$ not containing 0 such that $\mu(F)>0$ and $m\left(F^{\prime}\right)$ is contained in the cone generated by $K$ for all $F^{\prime} \subseteq F$.

Hypothesis 3 is the traditional type of hypothesis which has been used in previous Radon-Nikodym theorems for vector-valued measures. We discuss these previous theorems below. But it is not obvious that hypothesis (3) is satisfied by every realvalued measure, and so, if hypothesis (3) is used, the classical Radon-Nikodym theorem is not an immediate consequence of the Main Theorem. Hypothesis ( $\left.3^{\prime}\right)$, on the other hand, is clearly satisfied by every real-valued measure, or, more generally, by every measure with values in a finite dimensional Banach space (let $K$ be the entire surface of the unit ball of $B$ ). Also, hypothesis $\left(3^{\prime}\right)$ can be interpreted geometrically as follows: the role of $K$ is to specify a solid angle in $B$ (in fact $K$ can be taken to be a compact subset of the surface of the unit sphere in $B$, if desired), and hypothesis $\left(3^{\prime}\right)$ says that locally there are sets of positive measure on subsets of which the directions of the values of $m$ lie in this solid angle.

We feel that the methods used in proving the Main Theorem are at least as interesting as the theorem itself. Our proof is entirely elementary in the sense that we use only very basic facts about Banach spaces. In particular, we do not use

Received by the editors March 10, 1966 and, in revised form, July 20, 1966.

(1) The major portion of this research was supported by National Science Foundation Grant GP-4020. 
linear functionals, much less the Hahn-Banach theorem (we do use the KreinMilman theorem for norm compact subsets of a Banach space, but we give an elementary proof of this theorem for this special case $(\S 4)$ ). The crux of our proof is an analogue for vector-valued measures (Theorems 2.4 and 2.7) of the Hahn decomposition theorem for real-valued measures. This result may be of independent interest.

Finally, we indicate how the Main Theorem can be used to give new proofs of several previously obtained Radon-Nikodym theorems, even for weakly integrable functions with values in a locally convex topological vector space. This is done by showing that if $K$ is a subset of a locally convex space whose relative topology is compact and has a countable base, then a norm can be defined on the linear manifold spanned by $K$ in such a way that on $K$ the norm topology coincides with the original topology (Theorem 5.2). This result may be useful in other situations in which it is desired to apply results about Banach spaces to locally convex spaces.

We now discuss briefly the relation between the hypotheses of the Main Theorem and the hypotheses of Radon-Nikodym theorems obtained previously. Hypotheses (1) and (2) and the fact that the measure space must be assumed $\sigma$-finite [10, p. 131, Exercise 8] are familiar from the real-valued case, although for vector-valued measures hypothesis (2) is considerably stronger than just the requirement that the measure be finite. As has been mentioned, hypothesis (3) is the traditional type of hypothesis which has been used in previous Radon-Nikodym theorems for vector-valued measures. The key hypothesis of the Dunford-Pettis theorem [7, Theorem 2.1.1] is equivalent to the assumption that $A_{X}(m)$ is a bounded, and so relatively $w^{*}$ compact, subset of the dual of a separable Banach space. In Phillips' theorem [13, p. 130] it is assumed that $A_{X}(m)$ is a relatively weakly compact subset of a Banach space. A similar condition is used by Rao [14]. In the theorems of Dunford-Schwartz [8, p. 499] and Bourbaki [3, p. 46, Corollary 3] the main hypothesis is essentially that $A_{X}(m)$ is a relatively compact subset of a locally convex space satisfying certain separability hypotheses. A similar condition has been used by Dubins [5, p. 291]. Dieudonné [4], in giving a generalization of the Dunford-Pettis theorem, seems to have been the first to have required compactness of $A_{E}$ only for certain $E$ (although Phillips had considered a somewhat analogous condition). Metivier [12, p. 334] has generalized the theorem of Bourbaki in much the same way. We have not seen any analogues of hypothesis $\left(3^{\prime}\right)$.

Because the hypotheses of the theorems of Dunford-Pettis, Dieudonné, DunfordSchwartz, and Bourbaki explicitly include separability assumptions, and because separability is a consequence (nontrivial) of the hypotheses of Phillips' theorem (see [9, p. 426] or [3, p. 95, Exercise 24] $\left({ }^{2}\right)$ ), our Main Theorem can be applied, via Theorem 5.2 mentioned above, to give new proofs of these theorems. Of

$\left({ }^{2}\right)$ Added in proof. See also M. Metivier, Martingales à valeurs vectorielles; application à la dérivation, Ann. Inst. Fourier (Grenoble) (to appear). 
course conversely, Phillips' theorem could be used to prove our Main Theorem, but such a proof could hardly be called elementary in the sense which we have been using. More recently A. and C. Ionescu Tulcea [16], [17] have shown, by quite delicate arguments depending essentially on Zorn's Lemma, that the separability assumptions in the theorems mentioned above are not needed. We consider it very unlikely that our Main Theorem can be used to prove these theorems in the absence of separability assumptions. Also our results seem to have little in common with the Radon-Nikodym theorem of Rickart [15] in which the objects to be integrated are no longer single-valued functions.

Our results are organized in the following way. In $\$ 1$ we obtain certain properties of measurable and integrable functions in order to show the necessity of the hypotheses of the Main Theorem and also to motivate certain points in the proof of sufficiency. $\$ 2$ is devoted to the statement and proof of our analogue of the Hahn decomposition theorem. In $\$ 3$ the proof of the Main Theorem is given, and hypotheses (3) and ( $\left.3^{\prime}\right)$ are shown to be equivalent. The elementary proof of the Kreĭn-Milman theorem for norm compact sets is given in $\S 4$. We indicate in $\S 5$ how our Main Theorem can be used to prove previously obtained Radon-Nikodym theorems, as mentioned above. Finally, in $\S 6$ we collect a few examples pertinent to our results. In particular these examples show the independence of the hypotheses of the Main Theorem.

In a future paper we hope to be able to use techniques analogous to those introduced here to give a direct proof of a slight generalization of the RadonNikodym theorem of Phillips $\left({ }^{3}\right)$.

1. Measurable and integrable functions. Let $(X, S, \mu)$ be a $\sigma$-finite measure space [10], and let $B$ be a Banach space. We use the following definitions. A $B$ valued function, $f$, on $X$ is measurable [2] if it is the pointwise limit a.e. of a sequence of $B$-valued simple measurable functions, and $f$ is (Bochner) integrable if it is the limit a.e. of a mean Cauchy sequence of simple integrable functions [6]. There is no difficulty in carrying out the development of Bochner integration given in [8] using these definitions, and in the slightly more general setting of a $\sigma$-finite rather than totally $\sigma$-finite measure space.

However, since measurable functions and finite measures are carried on measurable sets, in order to prove the Main Theorem it is sufficient to do so for the case in which $(X, S, \mu)$ is totally $\sigma$-finite. Also, there is no loss of generality in assuming that $(X, S, \mu)$ is complete. Since the exposition becomes slightly simpler if we assume that $(X, S, \mu)$ is totally $\sigma$-finite and complete, we will make this assumption throughout, although we will state some of the more important results in the more general setting.

$\left(^{3}\right)$ Added in proof. Our not entirely sucessful attempt appears in "Dentable subsets of Banach spaces, with application to a Randon-Nikodym theorem," in Functional analysis, Thompson, Washington, D.C., 1967. 
Many of the results in this section are known, in one form or another, but we have not seen elementary proofs for all of them. All of our proofs are elementary, in the sense used in the introduction.

All measurable and integrable functions in this section are assumed to have values in $B$.

Proposition 1.1. If $f$ is a measurable function, then $f$ is locally almost compact valued, that is, given $E \in S$ with $\mu(E)<\infty$, and given $\varepsilon>0$, there is an $F \in S, F \subseteq E$ such that $\mu(E-F)<\varepsilon$ and $f(F)$ is a (norm) relatively compact subset of $B$.

Proof. Let $f_{n}$ be a sequence of simple measurable functions converging to $f$ a.e. By Egoroff's theorem [8, p. 149] $f_{n}$ converges to $f$ almost uniformly on $E$, that is, there is an $F \in S, F \subseteq E$ such that $\mu(E-F)<\varepsilon$ and $f_{n}$ converges to $f$ uniformly on $F$. We show that $f(F)$ is totally bounded. Given $r>0$, choose $n$ such that $\left\|f(x)-f_{n}(x)\right\|$ $\leqq r$ for $x \in F$. Let $b_{1} \cdots b_{k}$ be the elements of the range of $f_{n}$. Then it is clear that the balls of radius $r$ about $b_{1} \cdots b_{k}$ cover $f(F)$.

Definition 1.2. Let $f$ be a measurable function, and let $E \in S$. Then the essential range of $f$ restricted to $E, \operatorname{er}_{E}(f)$, is defined to be the set of those $b \in B$ such that for every $\varepsilon>0$ the measure of $\{x \in E:\|f(x)-b\|<\varepsilon\}$ is strictly positive.

We list some obvious properties of essential ranges.

Proposition 1.3. If $f$ and $g$ are measurable functions, and if $E, F \in S$, then

(1) $\mathrm{er}_{E}(f)$ is a closed subset of $B$,

(2) $\operatorname{er}_{E}(f)$ is contained in the closure of the range of $f$ restricted to $E$.

(3) If $f=g$ a.e. on $E$, then $\operatorname{er}_{E}(f)=\operatorname{er}_{E}(g)$.

(4) If $\mu(E)=0$, then $\operatorname{er}_{E}(f)$ is empty.

(5) If $F \subseteq E$, then $\operatorname{er}_{F}(f) \subseteq \operatorname{er}_{E}(f)$.

COROLlary 1.4. If $f$ is a measurable function, then $f$ is locally almost essentially compact valued, that is, given $E \in S$ with $\mu(E)<\infty$, and given $\varepsilon>0$, there is an $F \in S, F \subseteq E$ such that $\mu(E-F)<\varepsilon$ and $\operatorname{er}_{F}(f)$ is compact.

We remark that we have not yet shown that $\operatorname{er}_{E}(f)$ is not empty if $\mu(E)>0$. Hence the importance of

LEMMA 1.5. Let $f$ be a measurable function and let $E \in S$. If $\mu(E)>0$, then $\operatorname{er}_{E}(f)$ meets the range of $f$ restricted to $E$.

Proof. (This proof, simpler than our original proof, was found simultaneously by a number of my students.) By removing a suitable null set we can assume that $f(E)$ is separable. Suppose that $\operatorname{er}_{E}(f) \cap f(E)$ is empty. Then for each $x \in E$ there is an $\varepsilon_{x}>0$ such that

$$
\mu\left(\left\{y \in E:\|f(y)-f(x)\|<\varepsilon_{x}\right\}\right)=0 .
$$

The balls of center $f(x)$ and radius $\varepsilon_{x}$ cover $f(E)$, and so, since $f(E)$ is a separable 
metric space, there is a countable subcollection of these balls, with centers $f\left(x_{n}\right)$, which still cover $f(E)$. Then

so that $\mu(E)=0$.

$$
E \subseteq \bigcup_{n=1}^{\infty}\left\{y \in E:\left\|f(y)-f\left(x_{n}\right)\right\|<\varepsilon_{x_{n}}\right\}
$$

COROLlaRY 1.6. If $f$ is a measurable function, and $E \in S$, then

$$
N=\left\{x \in E: f(x) \notin \operatorname{er}_{E}(f)\right\}
$$

is a null set.

Proof. If $\mu(N)>0$ then by Lemma 1.5 there is an $x \in N$ such that $f(x) \in \operatorname{er}_{N}(f)$ $\subseteq \mathrm{er}_{E}(f)$ which contradicts the definition of $N$.

It is not difficult to use this result to prove

COROLlaRY 1.7. If $f$ is measurable and $E=\bigcup_{n=1}^{\infty} E_{n}, E_{n} \in S$, then

$$
\operatorname{er}_{E}(f)=\operatorname{closure}\left(\bigcup_{n=1}^{\infty} \operatorname{er}_{E_{n}}(f)\right) \text {. }
$$

LEMMA 1.8. Let $f$ and $g$ be measurable functions, and suppose that $\|f(x)-g(x)\| \leqq d$ a.e. on $E \in S$. Let $b \in \mathrm{er}_{E}(f)$. Then

$$
\text { distance }\left(b, \operatorname{er}_{E}(g)\right) \leqq d .
$$

Proof. Given $\varepsilon>0$, let

$$
F=\{x \in E:\|b-f(x)\|<\varepsilon, \text { and }\|f(x)-g(x)\| \leqq d\} .
$$

Then $\mu(F)>0$. By Lemma 1.5 there is a $y \in F$ such that $g(y) \in \operatorname{er}_{F}(g)$. But $\|b-g(y)\|<d+\varepsilon$. Since $\varepsilon$ is arbitrary, the proof is completed.

We will use the following notation. If $D \subseteq B$ then $c(D)$ will denote the convex hull of $D$ [8, p. 414], $\bar{c}(D)$ will denote the closed convex hull of $D$, and cone $(D)$ will denote the cone with vertex 0 generated by $D$. If $f$ is an integrable function then the indefinite integral of $f, \mu_{f}$, is the $B$-valued measure defined by

$$
\mu_{f}(E)=\int_{E} f d \mu, \quad E \in S
$$

For the basic properties of indefinite integrals we refer the reader to [8, p. 114].

Proposition 1.9 (THE MEAN-VAlUe THEOREM). If $f$ is an integrable function, and if $E \in S$ is such that $0<\mu(E)<\infty$, then

so that

$$
\cdot \mu_{f}(E) / \mu(E) \in \bar{c}\left(\operatorname{er}_{E}(f)\right),
$$

where

$$
A_{E}\left(\mu_{f}\right) \subseteq \bar{c}\left(\operatorname{er}_{E}(f)\right)
$$

$$
A_{E}\left(\mu_{f}\right)=\left\{\mu_{f}(F) / \mu(F): F \in S, F \subseteq E, 0<\mu(F)\right\},
$$

the average range of $\mu_{f}$ on $E$. 
Proof. Given $\varepsilon>0$ we show that the distance from $\mu_{f}(E) / \mu(E)$ to $\bar{c}\left(\operatorname{er}_{E}(f)\right)$ is less than $\varepsilon$. Since $\mu_{f}$ is absolutely $\mu$-continuous [8, p. 114], we can find $\delta>0$ such that if $\mu(F)<\delta$ then $\left\|\mu_{f}(F)\right\|<\mu(E) \varepsilon / 6$.

Let $f_{n}$ be a sequence of simple integrable functions converging to $f$ a.e., and so almost uniformly on $E$. Choose $F \in S, F \subseteq E$ such that $f_{n}$ converges uniformly to $f$ on $F, \mu(E-F)<\delta$, and $1 / \mu(F)<1 / \mu(E)+\varepsilon / 6\|f\|_{1}$. Then it is easily verified that

$$
\left\|\mu_{f}(E) / \mu(E)-\mu_{f}(F) / \mu(F)\right\|<\varepsilon / 3 .
$$

Thus it suffices to show that the distance from $\mu_{f}(F) / \mu(F)$ to $\bar{c}\left(\operatorname{er}_{F}(f)\right)$ is less than $2 \varepsilon / 3$.

Now choose $m$ such that $\left\|f(x)-f_{m}(x)\right\| \leqq \varepsilon / 4$ for $x \in F$. Then it is easily verified that

$$
\left\|\mu_{f}(F) / \mu(F)-\mu_{f_{m}}(F) / \mu(F)\right\|<\varepsilon / 3 .
$$

Thus it suffices to show that the distance from $\mu_{f_{m}}(F) / \mu(F)$ to $\bar{c}\left(\operatorname{er}_{F}(f)\right)$ is less than $\varepsilon / 3$.

Let $b_{1} \cdots b_{k}$ be the elements of $\operatorname{er}_{F}\left(f_{m}\right)$, so that on $F$

$$
f_{m}=\sum_{i=1}^{k} b_{i} \chi_{F_{i}} \quad \text { a.e., }
$$

where the $F_{i}$ are disjoint, $F=\bigcup_{i=1}^{k} F_{i}$, and $\mu\left(F_{i}\right)>0$ for each $i$. By Lemma 1.8 we can find $a_{i} \in \operatorname{er}_{F_{i}}(f)$ such that $\left\|b_{i}-a_{i}\right\|<\varepsilon / 3$ for each $i$. Then $\sum_{i=1}^{k}\left(\mu\left(F_{i}\right) / \mu(F)\right) a_{i}$ is in $\bar{c}\left(\operatorname{er}_{F}(f)\right)$ since $\sum_{i=1}^{k} \mu\left(F_{\imath}\right) / \mu(F)=1$. But it is easily verified that

$$
\left\|\mu_{f_{m}}(F) / \mu(F)-\sum_{i=1}^{k}\left(\mu\left(F_{i}\right) / \mu(F)\right) a_{i}\right\|<\varepsilon / 3 .
$$

Proposition 1.10. If $f$ is an integrable function, and if $E \in S$, then

$$
\operatorname{er}_{E}(f) \subseteq \bar{A}_{E}\left(\mu_{f}\right)
$$

(the closure of $A_{E}\left(\mu_{f}\right)$ ).

Proof. Let $b \in \operatorname{er}_{E}(f)$. Then, given $\varepsilon>0$, let $F=\{x \in E:\|b-f(x)\|<\varepsilon\}$, so that $\mu(F)>0$. Choose $F_{1} \in S, F_{1} \subseteq F$, such that $0<\mu\left(F_{1}\right)<\infty$. Then $\bar{c}\left(\operatorname{er}_{F_{1}}(f)\right)$ is contained in the ball of radius $\varepsilon$ about $b$. But by Proposition 1.9, $\mu_{f}\left(F_{1}\right) / \mu\left(F_{1}\right)$ $\in \bar{c}\left(\operatorname{er}_{F_{1}}(f)\right)$, and so $\left\|b-\mu_{f}\left(F_{1}\right) / \mu\left(F_{1}\right)\right\| \leqq \varepsilon$.

Corollary 1.11. If $f$ is an integrable function, and $E \in S$, then

$$
\bar{c}\left(A_{E}\left(\mu_{f}\right)\right)=\bar{c}\left(\operatorname{er}_{E}(f)\right) \text {. }
$$

We remark that if $\operatorname{er}_{E}(f)$ is compact, then it follows from the Kreln-Milman theorem that every extreme point of $\bar{c}\left(A_{E}\left(\mu_{f}\right)\right)$ is an element of $\operatorname{er}_{E}(f)$. This observation is the motivation for a key step in the proof of our decomposition theorem. 
We are now in a position to prove the necessity of the hypotheses of our Main Theorem.

Proposition 1.12. If $f$ is an integrable function, then $\mu_{f}$ satisfies hypotheses (1), (2), (3) and (3') of the Main Theorem. In fact, hypothesis (3) can be strengthened to

(3a) locally $m$ almost has compact average range, that is, given $E \in S$ with $\mu(E)<\infty$, and given $\varepsilon>0$, there is an $F \subseteq E$ such that $\mu(E-F)<\varepsilon$ and $A_{F}(m)$ is relatively compact.

Proof. The fact that $\mu_{f}$ satisfies hypotheses (1) and (2) of the Main Theorem follows from Theorem 20 on p. 114 of [8]. With respect to hypothesis (3a), if $E \in S$ with $\mu(E)<\infty$, and if $\varepsilon>0$ are given, then by Corollary 1.4 there is an $F \in S, F \subseteq E$, such that $\mu(E-F)<\varepsilon$ and $\operatorname{er}_{F}(f)$ is compact. Then $\bar{c}\left(\operatorname{er}_{F}(f)\right)$ is also compact (see [8, p. 416] for an elementary proof). But by Proposition $1.9 A_{F}\left(\mu_{f}\right)$ $\subseteq \bar{c}\left(\operatorname{er}_{F}(f)\right)$, and so $A_{F}\left(\mu_{f}\right)$ is relatively compact.

Finally, we show that $\mu_{f}$ satisfies hypothesis ( $\left.3^{\prime}\right)$. Given $E \in S$ with $0<\mu(E)<\infty$, choose $F_{0} \subseteq E$ as above so that $\operatorname{er}_{F_{0}}(f)$ is compact and $\mu\left(F_{0}\right)>0$. If $\operatorname{er}_{F_{0}}(f)=\{0\}$ then the range of $\mu_{f}$ on $F_{0}$ is $\{0\}$ which is contained in the cone generated by any single point. Otherwise, choose $b \in \operatorname{er}_{F_{0}}(f), b \neq 0$, and let $\delta=\|b\| / 2$. Let

$$
F=\left\{x \in F_{0}:\|f(x)-b\|<\delta\right\},
$$

so that $\mu(F)>0$, and let $K=\bar{c}\left(\operatorname{er}_{F}(f)\right)$, so that $K$ is compact and convex, and $0 \notin K$. Then $\mu_{f}\left(F^{\prime}\right) / \mu\left(F^{\prime}\right)$ is in $K$ for all $F^{\prime} \subseteq F, \mu\left(F^{\prime}\right)>0$, and so $\mu_{f}\left(F^{\prime}\right)$ is in cone $(K)$ for all $F^{\prime} \subseteq F$.

The proof that the hypotheses of the Main Theorem are sufficient is partly motivated by Theorem 18 of [8, p. 297]. We will prove here the part of this theorem which we need, but for the more general case of $B$-valued functions, and also because the proof in [8] is incorrect (the second inequality in line 4, p. 298, is false).

Following [8, p. 297] let $\Pi$ denote the set of all collections, $\pi$, consisting of a finite number of disjoint elements of $S$ with strictly positive finite measure. Then $\Pi$ is essentially a directed set when $\pi_{1} \geqq \pi$ is defined to mean that every element of $\pi$ is, except for a null set, the union of elements of $\pi_{1}$.

For each $\pi \in \Pi$, and each measurable function, $f$, which is integrable on sets of finite measure, define a function, $f_{\pi}$, by

$$
f_{\pi}=\sum_{E \in \pi}\left(\mu_{f}(E) / \mu(E)\right) \chi_{E}
$$

where $\mu_{f}(E)=\int_{E} f d \mu$. Each $f_{\pi}$ is a simple integrable function, and thus is in $L^{p}(X, S, \mu, B)$ for $1 \leqq p<\infty$.

Proposition 1.13. Let $1 \leqq p<\infty$. Then for each $f \in L^{p}(X, S, \mu, B)$ the net $\left\{f_{\pi}\right\}_{\pi \in \Pi}$ converges to $f$ in p-norm. 
Proof. Let $U_{\pi}$ be the linear operator carrying $L^{p}$ into itself defined by $U_{\pi}(f)=f_{\pi}$. We first show that $\left\|U_{\pi}\right\|_{p} \leqq 1$. If $f \in L^{p}$, then

$$
\left\|U_{\pi}(f)\right\|_{p}^{p}=\sum_{E \in \pi}\left\|\int_{E} f d \mu\right\|^{p}(\mu(E))^{1-p}
$$

which by Hölder's inequality on each $E \in \pi$ is

$$
\begin{aligned}
& \leqq \sum_{E \in \pi}\left[\left(\int_{E} d \mu\right)^{1 / q}\left(\int_{E}\|f(x)\|^{p} d \mu(x)\right)^{1 / p}\right]^{p}(\mu(E))^{1-p} \\
& =\sum_{E \in \pi} \int_{E}\|f(x)\|^{p} d \mu(x) \leqq\|f\|_{p}^{p},
\end{aligned}
$$

where $1 / p+1 / q=1$. Thus $\left\|U_{\pi}\right\|_{p} \leqq 1$.

Now if $f$ is a simple function in $L^{p}$, so that $f=\sum_{i=1}^{k} b_{i} \chi_{E_{i}}$ a.e., where the $E_{i}$ are disjoint sets of strictly positive finite measure, and if we let $\pi=\left\{E_{1} \cdots E_{k}\right\}$, then it is easily checked that $U_{\pi_{1}}(f)=f$ a.e. for $\pi_{1} \geqq \pi$. Finally, if $f$ is any element of $L^{p}$, and if $\varepsilon>0$ is given, let $g$ be a simple integrable function such that $\|f-g\|_{p}<\varepsilon / 2$. Let $g=\sum_{i=1}^{k} b_{i} \chi_{E_{i}}$ a.e. where the $E_{i}$ are disjoint sets of strictly positive finite measure, and let $\pi=\left\{E_{1} \cdots E_{k}\right\}$. Then

$$
\left\|f-f_{\pi_{1}}\right\|_{p} \leqq\|f-g\|_{p}+\left\|U_{\pi_{1}}(g-f)\right\|_{p}<\varepsilon
$$

for $\pi_{1}>\pi$.

Motivated by this result, the proof of the sufficiency of the conditions of our Main Theorem consists of showing that if for each $\pi \in \Pi$ we define a simple integrable function $f_{\pi}$ by

$$
f_{\pi}=\sum_{E \in \pi}(m(E) / \mu(E)) \chi_{E}
$$

then the net $\left\{f_{\pi}\right\}$ is a mean Cauchy net. It will then follow easily that if $f$ is a limit of $\left\{f_{\pi}\right\}$, then $m=\mu_{f}$.

To show that $\left\{f_{\pi}\right\}$ is a Cauchy net we must show that given $\varepsilon>0$ there is a $\pi \in \Pi$ such that if $\pi_{1}>\pi$ then $\left\|f_{\pi_{1}}-f_{\pi}\right\|_{1}<\varepsilon$. If this statement is analysed, it can be seen that, roughly speaking, each $E \in \pi$ must have the property that the vectors $m(F) / \mu(F)$ all stay close together as $F$ varies inside $E$. Our Decomposition Theorem states that if $m$ satisfies suitable conditions, involving hypothesis (3), then a $\pi \in \Pi$ whose elements have this property can be found.

2. The Decomposition Theorem. The key concept used in the Hahn decomposition theorem is the concept of sets which are (purely) positive or (purely) negative $[10$, p. 120] with respect to a given measure. These are defined in terms of the order relation on the real numbers. We will replace this concept of pure set by one defined in terms of convex cones in Banach spaces. Also, we will need to consider sets which are pure for a given $B$-valued measure relative to a given positive 
measure, this concept being defined in terms of general convex sets in Banach spaces.

Definition 2.1. Let $(X, S, \mu)$ be a $\sigma$-finite measure space, and let $m$ be a $B$ valued measure on $S$. If $K$ is a closed convex cone in $B$ (with vertex 0 ) then a measurable set $E$ is called $K$-pure for $m$ if $m(F) \in K$ for all $F \subseteq E$. If $m$ is $\mu$-continuous and if $K$ is any closed convex subset of $B$, then a measurable set $E$ will be called $K$-pure for $m$ relative to $\mu$, or $(K, \mu)$-pure, if $m(F) / \mu(F) \in K$ for every $F \subseteq E$ with $0<\mu(F)<\infty$, that is, if $A_{E}(m) \subseteq K$.

It is clear that any subset of a $K$-pure (resp. $(K, \mu)$-pure) set is $K$-pure (resp. $(K, \mu)$-pure). The most important property of pure sets, which depends on the fact that $K$ is closed and convex, is

Proposition 2.2. Any countable union of $K$-pure (resp. $(K, \mu)$-pure) sets is a K-pure (resp. $(K, \mu)$-pure) set.

Proof. Let $E=\bigcup_{i=1}^{\infty} E_{i}$ where each $E_{i}$ is $K$-pure (resp. $(K, \mu)$-pure). Since the difference of $K$-pure (resp. $(K, \mu)$-pure) sets is clearly $K$-pure (resp. $(K, \mu)$-pure), it suffices to consider the case in which the $E_{i}$ are disjoint. Let $F \subseteq E$, with $0<\mu(F)$ $<\infty$ in the $(K, \mu)$-pure case. Then $F=\bigcup_{i=1}^{\infty} F \cap E_{i}$, this union being disjoint.

Then in the $K$-pure case $m(F)=\sum_{i=1}^{\infty} m\left(F \cap E_{i}\right)$ where $m\left(F \cap E_{i}\right) \in K$ for each $i$. Since $K$ is a closed convex cone, it follows that $m(F) \in K$.

In the $(K, \mu)$-pure case we have

$$
m(F) / \mu(F)=\sum_{i=1}^{\infty}\left(m\left(F \cap E_{i}\right) / \mu\left(F \cap E_{i}\right)\right)\left(\mu\left(F \cap E_{i}\right) / \mu(F)\right),
$$

(where 0 is understood for terms in which $\mu\left(F \cap E_{i}\right)=0$ ) and so $m(F) / \mu(F)$ is expressed as an infinite convex combination of elements of $K$, since

$$
\sum_{i=1}^{\infty} \mu\left(F \cap E_{i}\right) / \mu(F)=1 .
$$

Since $K$ is convex and closed, it follows that $m(F) / \mu(F)$ is itself in $K$.

The next result provides some additional motivation for the proofs of the Decomposition Theorem and the Main Theorem.

Proposition 2.3. Let $f$ be an integrable function, and let $K$ be a closed convex subset of $B$. Then $E \in S$ is $(K, \mu)$-pure for $\mu_{f}$ if and only if $\operatorname{er}_{E}(f) \subseteq K$.

Proof. If $\operatorname{er}_{E}(f) \subseteq K$ and if $F \subseteq E$ with $0<\mu(F)<\infty$, then

$$
\mu_{f}(F) / \mu(F) \in \bar{c}\left(\operatorname{er}_{F}(f)\right) \subseteq K .
$$

Conversely, suppose that $b \in \operatorname{er}_{E}(f)$ but $b \notin K$. Let $\varepsilon>0$ be smaller than the distance from $b$ to $K$, and let

$$
F=\{x \in E:\|f(x)-b\|<\varepsilon\}
$$


Then $\mu(F)>0$, and if $F_{1} \subseteq F$ with $0<\mu\left(F_{1}\right)<\infty$, then $\mu_{f}\left(F_{1}\right) / \mu\left(F_{1}\right)$ is in the ball of radius $\varepsilon$ about $b$, and so is not in $K$.

Decomposition Theorem 2.4. Let $(X, S, \mu)$ be a $\sigma$-finite measure space, and let $m$ be a B-valued measure on $S$ which is $\mu$-continuous. Let $E \in S$ and suppose that $\bar{A}_{E}(m)$ is compact. Let $\left\{U_{1} \cdots U_{n}\right\}$ be a collection of open convex subsets of $B$ which covers $\bar{A}_{E}(m)$. Then there is a collection, $\left\{E_{1} \cdots E_{n}\right\}$, of measurable sets whose union is $E$ such that $E_{i}$ is $\left(\bar{U}_{i}, \mu\right)$-pure for $1 \leqq i \leqq n$.

It is not evident that there are any nonnull $\left(\bar{U}_{i}, \mu\right)$-pure sets at all, and the crux of the proof of the theorem is the following lemma which asserts that there is at least one. The proof of the theorem will then follow by a simple exhaustion argument.

If $K$ is the closed ball of radius $\delta$ about the point $b \in B$, it will be convenient to speak of $(b, \delta)$-pure sets instead of $K$-pure sets relative to $\mu$. We recall that if $K$ is a subset of $B$, then a point of $K$ is called an extreme point of $K$ if it lies in the interior of no line segment contained in $K$.

Key Lemma 2.5. Let $m$ and $E$ satisfy the hypotheses of Theorem 2.4, and let $\delta>0$ be given. Then there is $a b \in \bar{A}_{E}(m)$ and an $F \in S, F \subseteq E$, such that $\mu(F)>0$ and $F$ is $(b, \delta)$-pure. In fact $b$ can be chosen to be any extreme point of $\bar{c}\left(A_{E}\right)$.

Proof. The proof depends on the Kreinn-Milman theorem for the case of norm compact subsets of a Banach space. An elementary proof of this theorem for this case is given in $\S 4$. Here we will just recall the statement of the theorem.

THEOREM 2.6 (KREǏN-MILMAN THEOREM FOR NORM COMPACT SETS). If $K$ is a norm compact subset of $B$, and if $K^{e}$ is the collection of extreme points of $K$, then $\bar{c}\left(K^{e}\right)$ $=\bar{c}(K)$. Furthermore, every extreme point of $\bar{c}(K)$ lies in $K^{e}$.

We continue with the proof of the Key Lemma. Since $\bar{A}_{E}$ is compact, so is $\bar{c}\left(A_{E}\right)\left[8\right.$, p. 416]. Let $b$ be any extreme point of $\bar{c}\left(A_{E}\right)$. From the comment following Corollary 1.11 it follows that if the Main Theorem could be used, that is, if it were known that $m$ is the indefinite integral of an integrable function $f$, then $b \in \operatorname{er}_{E}(f)$, and so $\{x \in E:\|f(x)-b\|<\delta\}$ would be a nonnull $(b, \delta)$-pure set for $m$ relative to $\mu$. Thus under the hypotheses of the present lemma we might expect to be able to find a nonnull $(b, \delta)$-pure set for this special choice of $b$.

Let $B(b, \delta)$ denote the ball of radius $\delta$ about $b$, and let $Q=\bar{c}\left(A_{E}-B(b, \delta)\right)$. By Theorem $2.6 b \notin Q$ but $b \in \bar{A}_{E}$. Then, since $Q$ is closed, we can find $F_{0} \subseteq E$ with $0<\mu\left(F_{0}\right)<\infty$ such that $m\left(F_{0}\right) / \mu\left(F_{0}\right) \in B(b, \delta)-Q$.

Suppose that $F_{0}$ is not $(b, \delta)$-pure. We sweep out the part of $F_{0}$ which is not $(b, \delta)$-pure as follows. Let $k_{1}$ be the smallest integer $\geqq 2$ for which there exists $E_{1} \subseteq F_{0}$ such that $\mu\left(E_{1}\right) \geqq 1 / k_{1}$ and $m\left(E_{1}\right) / \mu\left(E_{1}\right)$ is in $Q$. Let $F_{1}=F_{0}-E_{1}$ and suppose that $F_{1}$ is not $(b, \delta)$-pure. Let $k_{2}$ be the smallest integer $\geqq 2$ for which there exists $E_{2} \subseteq F_{1}$ such that $\mu\left(E_{2}\right) \geqq 1 / k_{2}$ and $m\left(E_{2}\right) / \mu\left(E_{2}\right)$ is in $Q$. Let $F_{2}=F_{1}-E_{2}$. Continuing 
in this way we obtain a sequence of disjoint subsets, $E_{n}$, of $F_{0}$ and a nondecreasing sequence of integers, $k_{n}$, with the property that for each $n, \mu\left(E_{n}\right) \geqq 1 / k_{n}, m\left(E_{n}\right) / \mu\left(E_{n}\right)$ is in $Q$, and if $E^{\prime} \subseteq F_{0}-\bigcup_{i=1}^{n} E_{i}$ and $m\left(E^{\prime}\right) / \mu\left(E^{\prime}\right)$ is in $Q$ then $\mu\left(E^{\prime}\right)<1 /\left(k_{n}-1\right)$. Since $F_{0}$ has finite measure, $k_{n}$ must converge to $\infty$.

Let $E_{0}=\bigcup_{n=1}^{\infty} E_{n}$. Then, since the $E_{n}$ are disjoint,

$$
m\left(E_{0}\right) / \mu\left(E_{0}\right)=\sum_{n=1}^{\infty}\left(m\left(E_{n}\right) / \mu\left(E_{n}\right)\right)\left(\mu\left(E_{n}\right) / \mu\left(E_{0}\right)\right) .
$$

Since $\sum_{n=1}^{\infty} \mu\left(E_{n}\right) / \mu\left(E_{0}\right)=1$, and $m\left(E_{n}\right) / \mu\left(E_{n}\right)$ is in $Q$ for each $n$, and since $Q$ is convex and closed, it follows that $m\left(E_{0}\right) / \mu\left(E_{0}\right)$ is in $Q$.

Let $F=F_{0}-E_{0}$. Then $F$ is $(b, \delta)$-pure, for if $F^{\prime} \subseteq F, \mu\left(F^{\prime}\right)>0$, and $m\left(F^{\prime}\right) / \mu\left(F^{\prime}\right)$ is in $Q$, then $F^{\prime} \subseteq F_{0}-\bigcup_{i=1}^{n} E_{i}$ for each $n$, and so $\mu\left(F^{\prime}\right)<1 /\left(k_{n}-1\right)$ for each $n$. Since $k_{n}$ converges to $\infty, \mu\left(F^{\prime}\right)=0$, so that no such $F^{\prime}$ exists.

Finally, we note that $\mu(F)>0$, for if $\mu(F)=0$, then $m(F)=0$, and so $m\left(F_{0}\right) / \mu\left(F_{0}\right)$ $=m\left(E_{0}\right) / \mu\left(E_{0}\right)$, which is in $Q$, and this contradicts the way in which $F_{0}$ was chosen.

Proof of Theorem 2.4. First suppose that $\mu(E)<\infty$. For each $i, 1 \leqq i \leqq n$, let

$$
a_{i}=\sup \left\{\mu(F): F \subseteq E \text { and } F \text { is }\left(\bar{U}_{i}, \mu\right) \text {-pure }\right\} .
$$

Then $a_{i}$ is finite since $\mu(E)<\infty$. Let $E_{i j}$ be a sequence of $\left(\bar{U}_{i}, \mu\right)$-pure subsets of $E$ such that $\lim _{j} \mu\left(E_{i j}\right)=a_{i}$, and let $E_{i}=\bigcup_{j=1}^{\infty} E_{i j}$. Then $E_{i}$ is a $\left(\bar{U}_{i}, \mu\right)$-pure set by Proposition 2.2, and, since it is clear that $\mu\left(E_{i}\right)=a_{i}$, it follows that $E_{i}$ is a $\left(\bar{U}_{i}, \mu\right)$ pure subset of maximal measure in $E$.

Let $F=E-\bigcup_{i=1}^{n} E_{i}$. We show that $\mu(F)=0$. Suppose that $\mu(F)>0$, and let $b$ be any extreme point of $\bar{c}\left(A_{F}\right)$. Since $b$ is in $\bar{A}_{F}, b$ is contained in $U_{i_{0}}$ for some $i_{0}$, so that we can choose $\delta>0$ such that $B(b, \delta)$ is contained in $U_{i_{0}}$. Then by the Key Lemma 2.5 there is an $F^{\prime} \subseteq F$ such that $\mu\left(F^{\prime}\right)>0$ and $F^{\prime}$ is $(b, \delta)$-pure and so $\left(\bar{U}_{i_{0}}, \mu\right)$-pure. Since $F^{\prime}$ is disjoint from $E_{i_{0}}$ this contradicts the maximality of $E_{i_{0}}$. Thus $\mu(F)=0$. Then we can adjoin $F$ to any $E_{i}$, so that $E=\bigcup_{i=1}^{n} E_{i}$, where $E_{i}$ is $\left(\bar{U}_{i}, \mu\right)$-pure for $1 \leqq i \leqq n$.

Now suppose that $E$ does not have finite measure. Then since $\mu$ is $\sigma$-finite, $E=\bigcup_{j=1}^{\infty} F_{j}$ where $\mu\left(F_{j}\right)<\infty$ for each $j$. Then by the first part of this proof we can find sets $E_{j i}, 1 \leqq i \leqq n$, such that $E_{j}=\bigcup_{i=1}^{n} E_{j i}$ for each $j$, and $E_{j i}$ is $\left(\bar{U}_{i}, \mu\right)$-pure for each $i$. Let $E_{i}=\bigcup_{j=1}^{\infty} E_{j i}$. Then $E_{i}$ is $\left(\bar{U}_{i}, \mu\right)$-pure for each $i$ by Proposition 2.2, and it is clear that $E=\bigcup_{i=1}^{n} E_{i}$.

While Theorem 2.4 is in the form which we need for the proof of the Main Theorem, it has the disadvantage that the usual Hahn decomposition theorem is not apparently a corollary. For this reason we now state the theorem in a form such that the Hahn decomposition theorem, at least for finite measures, is a corollary.

THeOREM 2.7. Let $m$ be a B-valued measure and let $E \in S$ be such that $|m|$ is totally $\sigma$-finite on $E$. Suppose that there is a compact subset, $K$, of $B$ not containing 0 such that the range of $m$ restricted to $E$ is contained in cone $(K)$. Let $\left\{U_{1} \cdots U_{n}\right\}$ be a collection of open convex subsets of $B$ which cover $K$. Then there is a collection 
$\left\{E_{1} \cdots E_{n}\right\}$ of measurable sets whose union is $E$ such that $E_{i}$ is cone $\left(\bar{U}_{i}\right)$-pure for $m$ for $1 \leqq i \leqq n$.

Proof. Let $\mu=|m|$. As in the proof of Theorem 2.4 it suffices to consider the case in which $\mu(E)<\infty$. Also by the argument used in the proof of Theorem 2.4 let $E_{1} \cdots E_{n}$ be subsets of $E$ of maximal measure such that $E_{i}$ is cone $\left(\bar{U}_{i}\right)$-pure for $1 \leqq i \leqq n$. Let $F=E-\bigcup_{i=1}^{n} E_{i}$.

We show that $\mu(F)=0$. Suppose on the contrary that $\mu(F)>0$. Then $A_{F}$ is not empty, and must contain points other than 0 , since otherwise $\mu(F)=|m|(F)=0$. Furthermore $\left\|m\left(F^{\prime}\right)\right\| \leqq|m|\left(F^{\prime}\right)=\mu\left(F^{\prime}\right)$ for any $F^{\prime} \in S$, so $A_{F}$ is contained in the unit ball of $B$. Let $1 / k$ be the distance from $K$ to 0 . Then

$$
A_{F} \subseteq \bar{c}(k K \cup\{0\})
$$

so that $\bar{c}\left(A_{F}\right)$ is compact. Since $A_{F}$ contains points other than $0, \bar{c}\left(A_{F}\right)$ must have a nonzero extreme point, say $b$. Since $b$ is in $\bar{A}_{F}, b$ is contained in the interior of cone $\left(U_{i_{0}}\right)$ for some $i_{0}$, so that we can choose $\delta>0$ such that $B(b, \delta)$ is contained in cone $\left(U_{i_{0}}\right)$. Then by the Key Lemma 2.5 there is an $F^{\prime} \subseteq F$ such that $\mu\left(F^{\prime}\right)>0$ and $F^{\prime}$ is $(b, \delta)$-pure relative to $\mu$. It follows that $F^{\prime}$ is cone $\left(U_{i_{0}}\right)$-pure, contradicting the maximality of $E_{i_{0}}$.

We remark that once the Main Theorem has been proven, Theorem 2.7 can be considerably strengthened in that the $U_{i}$ can be replaced by any countable collection of closed convex sets, $\left\{K_{i}\right\}$, whose union contains $K$. In fact, if $m$ is the indefinite integral of $f$ with respect to $\mu=|m|$ on $E$, then it suffices, except for a possible null set, to let $E_{i}=\left\{x \in E: f(x) \in \operatorname{cone}\left(K_{i}\right)\right\}$.

Corollary 2.8 (HAHN DECOMPOSITION THEOREM FOR FINITE MEASURES). If $m$ is a finite real measure, then there exist disjoint sets $E_{1}$ and $E_{2}$ whose union is $X$ such that $E_{1}$ is purely positive and $E_{2}$ is purely negative for $m$.

Proof. Let $K=\{1,-1\}$ and $U_{1}=(0,2), U_{2}=(-2,0)$. Then the only point which remains to be checked before applying Theorem 2.7 is that $|m|$ is finite. But this follows from [8, p. 97, Lemma 5]. (Of course it is important that we are defining $|m|$ by [8, p. 97, Definition 4] and not in terms of a Hahn decomposition as is done in [10].)

3. The proof of the Main Theorem. Assume first that $m$ satisfies hypotheses (1), (2) and (3a) (see Proposition 1.12). (At the end of this section we show that hypotheses (3) and ( $\left.3^{\prime}\right)$ are equivalent to (3a).) We now show that $\left\{f_{\pi}\right\}$, as defined at the end of $\S 1$, is a mean Cauchy net. Let $\varepsilon>0$ be given. Since $|m|$ is a finite measure, we can find $E \in S$ such that $\mu(E)<\infty$ and $|m|(X-E)<\varepsilon / 3$. Since $m$ is $\mu$-continuous, so is $|m|$. Then, since $|m|$ is a finite measure, it is absolutely $\mu$ continuous, $[10$, p. 125] so that we can find $\delta>0$ such that if $\mu(F)<\delta$ then $|m|(F)$ $<\varepsilon / 6$. Choose $E_{0} \subseteq E$ such that $\mu\left(E_{0}\right)<\delta$ and $\bar{A}_{E-E_{0}}$ is compact.

Let $b_{1} \cdots b_{n}$ be elements of $B$ which are $(\varepsilon / 6 \mu(E))$-dense in $\bar{A}_{E-E_{0}}$, that is, if $U_{i}$ 
denotes the open ball about $b_{i}$ of radius $\varepsilon / 6 \mu(E)$, then the $U_{i}$ cover $\bar{A}_{E-E_{0}}$. Then, applying the Decomposition Theorem 2.4, we can find disjoint sets $E_{1} \cdots E_{n}$ whose union is $E-E_{0}$ such that $E_{i}$ is $\left(b_{i}, \varepsilon / 6 \mu(E)\right)$-pure for each $i$. By eliminating those $E_{i}$ which are null sets, by eliminating the corresponding $b_{i}$, and by adjusting the remaining $E_{i}$ by null sets, we can assume that $\mu\left(E_{i}\right)>0$ for each $i$ (but the $b_{i}$ need no longer be $\varepsilon / 6 \mu(E)$-dense). Let $\pi_{0}=\left\{E_{i}: 0 \leqq i \leqq n\right\}$, or, if $\mu\left(E_{0}\right)=0$, let $\pi_{0}=\left\{E_{i}: 1 \leqq i \leqq n\right\}$, so that $\pi_{0} \in \Pi$.

We now show that if $\pi>\pi_{0}$, then $\left\|f_{\pi}-f_{\pi_{0}}\right\|_{1}<\varepsilon$. We will assume that $\mu\left(E_{0}\right)>0$, for it will be clear how the proof simplifies if $\mu\left(E_{0}\right)=0$. If $\pi>\pi_{0}$, then, except for possible null sets,

$$
\pi=\left\{F_{1} \cdots F_{k}\right\} \cup\left\{F_{i j}: 0 \leqq i \leqq n, 1 \leqq j \leqq k_{i}\right\}
$$

where the $F_{i}$ do not meet $E$ for $1 \leqq i \leqq k$, and $E_{i}=\bigcup_{j=1}^{k_{i}} F_{i j}$ for $0 \leqq i \leqq n$. Of course all the elements of $\pi$ are disjoint and have strictly positive measure. Then

$$
\begin{aligned}
\left\|f_{\pi}-f_{\pi_{0}}\right\|_{1}= & \int\left\|f_{\pi}(x)-f_{\pi_{0}}(x)\right\| d \mu(x) \\
= & \sum_{i=1}^{k}\left\|m\left(F_{i}\right)\right\|+\sum_{j=1}^{k_{0}}\left\|m\left(F_{0 j}\right) / \mu\left(F_{0 j}\right)-m\left(E_{0}\right) / \mu\left(E_{0}\right)\right\| \mu\left(F_{0 j}\right) \\
& +\sum_{i=1}^{n}\left\{\sum_{j=1}^{k_{i}}\left\|m\left(F_{i j}\right) / \mu\left(F_{i j}\right)-m\left(E_{i}\right) / \mu\left(E_{i}\right)\right\| \mu\left(F_{i j}\right)\right\} \\
\leqq & |m|(X-E)+\sum_{j=0}^{k_{0}}\left\|m\left(F_{0 j}\right)\right\|+\left\|m\left(E_{0}\right)\right\| \\
& +\sum_{i=1}^{n}\left\{\sum_{j=1}^{k_{i}}\left(\left\|m\left(F_{i j}\right) / \mu\left(F_{i j}\right)-b_{i}\right\|+\left\|b_{i}-m\left(E_{i}\right) / \mu\left(E_{i}\right)\right\|\right) \mu\left(F_{i j}\right)\right\} \\
\leqq & \varepsilon / 3+\varepsilon / 6+\varepsilon / 6+(\varepsilon / 3 \mu(E)) \mu\left(\bigcup_{i=1}^{n} E_{i}\right) \leqq \varepsilon .
\end{aligned}
$$

Thus the net $\left\{f_{\pi}\right\}$ is a mean Cauchy net, and so converges in mean to some element, $f$, of $L^{1}(X, S, \mu, B)$. In particular,

for every $E \in S$.

$$
\mu_{f}(E)=\int_{E} f d \mu=\lim _{\pi} \int_{E} f_{\pi} d \mu
$$

We now show that $m(E)=\mu_{f}(E)$ for all $E \in S$. Let $E \in S$. If $\mu(E)=0$ the result follows from the $\mu$-continuity of $m$. If $0<\mu(E)<\infty$, let $\pi_{0}=\{E\}$. Then it is easily checked that

whenever $\pi \geqq \pi_{0}$, and so

$$
\int_{E} f_{\pi} d \mu=m(E)
$$

$$
\mu_{f}(E)=\lim _{\pi} \int_{E} f_{\pi} d \mu=m(E) .
$$

The result when $\mu(E)=\infty$ then follows from the $\sigma$-finiteness of $\mu$. 
We now show that hypotheses (3) and $\left(3^{\prime}\right)$ are equivalent to hypothesis (3a) in the presence of hypotheses (1) and (2). Clearly hypothesis (3a) implies hypothesis (3). Also, if $m$ satisfies hypothesis (3a), then, by the part of the Main Theorem proved so far, $m=\mu_{f}$ for some Bochner integrable $f$, and so $m$ satisfies hypothesis $\left(3^{\prime}\right)$ by Proposition 1.12 (a direct proof can also be given).

Now let $m$ satisfy hypothesis ( $\left.3^{\prime}\right)$. Then we show that $m$ satisfies hypothesis (3). Given $E \in S$ with $0<\mu(E)<\infty$, choose $F_{0} \subseteq E$ and compact $K \subseteq B$ not containing 0 such that $\mu\left(F_{0}\right)>0$ and the range of $m$ on $F_{0}$ is contained in cone $(K)$. Choose a constant $c$ large enough so that the measure $c \mu-|m|$ is not purely negative on $F_{0}$, and let $F$ be the purely positive part of a Hahn decomposition (Corollary 2.8) of $c \mu-|m|$. Then $\mu(F)>0$, and $\left\|m\left(F^{\prime}\right) / \mu\left(F^{\prime}\right)\right\|<c$ for $F^{\prime} \subseteq F$ and $\mu\left(F^{\prime}\right)>0$. It follows that if $1 / k$ is the distance from $K$ to 0 , then $m\left(F^{\prime}\right) / \mu\left(F^{\prime}\right)$ is in $\bar{c}(c k K \cup\{0\})$ which is compact, and so hypothesis ( 3 ) is satisfied.

It remains to show that if $m$ satisfies hypothesis (3), then $m$ satisfies hypothesis (3a). First we make the easily checked observation that $A_{E \cup F} \subseteq c\left(A_{E} \cup A_{F}\right)$ for any $E, F \in S$, so that $\bar{A}_{E \cup F}$ is compact if $\bar{A}_{E}$ and $\bar{A}_{F}$ are. Now let $m$ satisfy hypothesis (3), and let $E \in S$ with $\mu(E)<\infty$ be given. Let

$$
a=\sup \left\{\mu(F): F \subseteq E \text { and } \bar{A}_{F} \text { is compact }\right\} .
$$

It suffices to show that $a=\mu(E)$. Let $\left\{F_{i}\right\}$ be a sequence of subsets of $E$ such that $\mu\left(F_{i}\right) \rightarrow a$ and $\bar{A}_{F_{i}}$ is compact. By the observation made above we can assume that the $F_{i}$ are increasing. Let $E_{0}=\bigcup F_{i}$, so that $\mu\left(E_{0}\right)=a$. If $a<\mu(E)$, then $\mu\left(E-E_{0}\right)>0$, so that by hypothesis (3) there is an $F_{0} \subseteq E-E_{0}$ such that $\mu\left(F_{0}\right)>0$ and $\bar{A}_{F_{0}}$ is compact. Then $\bar{A}_{F_{i} \cup F_{0}}$ is compact, and $\mu\left(F_{i} \cup F_{0}\right) \rightarrow a+\mu\left(F_{0}\right)$, contradicting the definition of $a$.

4. The Krein-Milman theorem. We now supply our elementary proof of Theorem 2.6. The crux of the matter is to show that there exists even one extreme point.

LeMma 4.1. Let $K$ be a norm compact subset of $B$. Then $K$ has at least one extreme point.

Proof. Since $K$ is a compact metric space, we can find a sequence, $\left\{b_{n}\right\}$, which is dense in $K$. We define subsets, $K_{n}$, of $K$ by induction. First, let $K_{0}=K$. Then, if $K_{n-1}$ has been defined, we define $K_{n}$ by

$$
K_{n}=\left\{x \in K_{n-1}:\left\|x-b_{n}\right\|=\sup \left\{\left\|y-b_{n}\right\|: y \in K_{n-1}\right\}\right\}
$$

A simple compactness argument shows that $K_{n}$ is nonempty. Also, $K_{n}$ is clearly closed and so compact, and $K_{n} \subseteq K_{n-1}$. Let $K^{\prime}=\bigcap_{n=1}^{\infty} K_{n}$. Then $K^{\prime}$ is not empty, since $K$ is compact. We show that $K^{\prime}$ contains exactly one point, and that this point is an extreme point of $K$. 
Suppose that $K^{\prime}$ contained two distinct points, $c$ and $c^{\prime}$. Since $\left\{b_{n}\right\}$ is dense in $K$, there is an $n$ such that

$$
\left\|c^{\prime}-b_{n}\right\|<\left\|c-b_{n}\right\|
$$

Then, since $c \in K_{n-1}$,

$$
\left\|c^{\prime}-b_{n}\right\|<\sup \left\{\left\|y-b_{n}\right\|: y \in K_{n-1}\right\},
$$

and so $c^{\prime} \notin K_{n}$, which contradicts the assumption that $c^{\prime} \in K^{\prime}$.

Let $e$ be the unique point in $K^{\prime}$, and suppose that $e$ is not an extreme point of $K$, so that

$$
e=t c_{1}+(1-t) c_{2}, \quad 0<t<1,
$$

for some $c_{1}, c_{2} \in K$. Let $n$ be the smallest integer such that either $c_{1}$ or $c_{2}$ is not in $K_{n}$, so that $c_{1}$ and $c_{2}$ are both in $K_{n-1}$. Then

$$
\left\|c_{i}-b_{n}\right\| \leqq \sup \left\{\left\|y-b_{n}\right\|: y \in K_{n-1}\right\}, \quad i=1,2,
$$

with strict inequality holding for $i=1$ or 2 . Then

$$
\begin{aligned}
\left\|e-b_{n}\right\| & \leqq t\left\|c_{1}-b_{n}\right\|+(1-t)\left\|c_{2}-b_{n}\right\| \\
& <\sup \left\{\left\|y-b_{n}\right\|: y \in K_{n-1}\right\},
\end{aligned}
$$

which contradicts the fact that $e \in K_{n}$. Thus $e$ must be an extreme point of $K$.

Proof of Theorem 2.6. If $K^{e}$ is the set of all extreme points of $K$, it is clear that $\bar{c}\left(K^{e}\right) \subseteq \bar{c}(K)$. Suppose that $\bar{c}\left(K^{e}\right) \neq \bar{c}(K)$, so that $K \nsubseteq \bar{c}\left(K^{e}\right)$. Let

$$
d=\sup \left\{\operatorname{distance}\left(y, \bar{c}\left(K^{e}\right)\right): y \in K\right\},
$$

so that $d>0$, and let

$$
K^{\prime}=\left\{x \in K: \operatorname{distance}\left(x, \bar{c}\left(K^{e}\right)\right)=d\right\} .
$$

Then $K^{\prime}$ is nonempty, compact, and disjoint from $\bar{c}\left(K^{e}\right)$.

Then $K^{\prime}$ has an extreme point, $e$, by Lemma 4.1. We show that $e$ is also an extreme point of $K$, contradicting the fact that $K^{e}$ contains all the extreme points of $K$. Suppose that $e$ is not an extreme point of $K$, so that

$$
e=t b_{1}+(1-t) b_{2}, \quad 0<t<1,
$$

for some $b_{1}, b_{2} \in K$. Since $e$ is an extreme point of $K^{\prime}$, at least one of $b_{1}, b_{2}$ is not in $K^{\prime}$, say $b_{1}$, so that distance $\left(b_{1}, \bar{c}\left(K^{e}\right)\right)<d$. By the compactness of $\bar{c}\left(K^{e}\right)$ there exist $c_{1}, c_{2} \in \bar{c}\left(K^{e}\right)$ such that

$$
\left\|b_{i}-c_{i}\right\|=\operatorname{distance}\left(b_{i}, \bar{c}\left(K^{e}\right)\right), \quad i=1,2 .
$$

In particular $\left\|b_{1}-c_{1}\right\|<d$ ar: $\left\|b_{2}-c_{2}\right\| \leqq d$. Let $c=t c_{1}+(1-t) c_{2}$. Then $c \in \bar{c}\left(K^{e}\right)$, and

$$
\|e-c\| \leqq t\left\|b_{1}-c_{1}\right\|+(1-t)\left\|b_{2}-c_{2}\right\|<d,
$$

contradicting the fact that $e \in K^{\prime}$. 
The final part of Theorem 2.6 has a standard elementary proof. See for example [8, p. 440, Lemma 5].

5. Other Radon-Nikodym theorems. In this section we indicate how our Main Theorem can be used to give new proofs of the Radon-Nikodym theorems of Dunford and Pettis [7], Phillips [13, p. 134], Dieudonné [4], Bourbaki [3, p. 46], and (with separability hypotheses) Metivier [12, p. 334]. We will not actually supply proofs of all these theorems here, but instead we will give a proof of a version of Theorem 2 of [8, p. 499]. Using this proof as a model, the reader may supply his own proofs of the above mentioned theorems.

We recall that if $V$ is a locally convex topological vector space, with dual space $V^{*}$, and if $(X, S, \mu)$ is a positive $\sigma$-finite measure space, then a function, $f$, from $X$ to $V$ is said to be weakly measurable if $\langle f(\cdot), z\rangle$ is measurable for all $z \in V^{*}$, and $f$ is said to be weakly integrable if $\langle f(\cdot), z\rangle$ is integrable for all $z \in V^{*}$ (where $\langle$,$\rangle denotes the dual pairing between V$ and $V^{*}$ ).

We now state Theorem 2 of [8, p. 499] explicitly in the form of a RadonNikodym theorem.

THeOREM 5.1. Let $(X, S, \mu)$ be a positive finite measure space, and let $V$ be a locally convex topological vector space. Let $m$ be a $V$-valued measure on $S$ which is $\mu$-continuous, and suppose that $\bar{A}_{X}(m)$ is a compact subset of $V$ whose relative topology has a countable base. Then there is a weakly integrable function, $f$, whose range is contained in $\bar{A}_{X}$, such that

$$
\langle m(E), z\rangle=\int_{E}\langle f(x), z\rangle d \mu(x)
$$

for all $z \in V^{*}$ and $E \in S$. That is, $m$ is the weak indefinite integral of $f$.

We have stated (and will prove) this theorem only for locally convex spaces. The reader will have no difficulty in supplying the few extra details needed to extend the theorem to the more general situation considered in [8].

Our proof of Theorem 5.1 (and the other Radon-Nikodym theorems mentioned above) depends on the following theorem, which enables us to apply to locally convex spaces the results we have obtained about Banach spaces.

THeOREM 5.2. Let $V$ be a locally convex topological vector space, and let $K$ be a compact subset of $V$. Let $K^{\prime}$ be the closed circled convex extension of $K$ [11, p. 14], and let $W$ be the linear manifold spanned by $K^{\prime}$. Then a norm can be defined on $W$ such that on $K^{\prime}$ the norm topology coincides with the original topology if and only if the original topology on $K$ has a countable base.

Proof. Since the topology of a compact metric space has a countable base, it is clear that if the original topology on $K$ coincides with a norm topology, then it has a countable base.

To prove the converse we first note that it suffices to do so in the case in which 
$V$ is complete $\left[11\right.$, p. 62]. For let $V^{c}$ be the completion of $V$. Then the relative topology of $K$ in $V^{c}$ will still be compact and have a countable base. Let $K^{\prime \prime}$ be the closed circled convex extension of $K$ in $V^{c}$, and let $W^{\prime \prime}$ be the linear manifold in $V^{c}$ spanned by $K^{\prime \prime}$. Then $K^{\prime} \subseteq K^{\prime \prime}$ and $W \subseteq W^{\prime \prime}$, and the topology of $K^{\prime}$ is just the relative topology from $K^{\prime \prime}$. If a norm can be defined on $W^{\prime \prime}$ such that on $K^{\prime \prime}$ the norm topology coincides with the original topology, then the restriction of this norm to $W$ will have the desired property that on $K^{\prime}$ the norm topology coincides with the original topology.

Now assume that $V$ is complete, so that $K^{\prime}$ is compact [11, p. 113]. Let $C(K)$ be the Banach space of all continuous scalar-valued functions on $K$. By Lemma 4 of [8, p. 501] $C(K)$ is separable, since the topology of $K$ has a countable base. Each element of $V^{*}$ can be viewed as an element of $C(K)$. Since any subset of a separable metric space is separable, there is a sequence $\left\{z_{n}\right\}$ of elements of $V^{*}$, none of which is identically zero on $K$, such that for any $z \in V^{*}$ and any $\varepsilon>0$ there is an $n$ such that

$$
\left|\left\langle v, z-z_{n}\right\rangle\right|<\varepsilon \text { for all } v \in K \text {. }
$$

Since $z-z_{n}$ is linear, this inequality persists for all $v \in K^{\prime}$.

Since $K^{\prime}$ is compact, each $z_{n}$ is bounded on $K^{\prime}$. For each $n$ let

$$
\left|z_{n}\right|=\sup \left\{\left|\left\langle v, z_{n}\right\rangle\right|: v \in K^{\prime}\right\}
$$

Note that the $z_{n}$ were chosen so that $\left|z_{n}\right|>0$ for each $n$.

Define a function, \|\| , on $W$ by

$$
\|w\|=\sum_{n=1}^{\infty} 2^{-n}\left|z_{n}\right|^{-1}\left|\left\langle w, z_{n}\right\rangle\right| .
$$

If $w \in K^{\prime}$, then $\left|\left\langle w, z_{n}\right\rangle\right| \leqq\left|z_{n}\right|$ for each $n$, so the sum converges. Since $K^{\prime}$ is circled and convex, each element of $W$ is just a scalar multiple of an element of $K^{\prime}$, so the sum converges for each element of $W$.

It is then clear that \|\| is a seminorm on $W$. We now show that it is a norm. If $w \in K^{\prime}$ and $w \neq 0$, then by the Hahn-Banach theorem there is a $z \in \bar{V}$ such that $|\langle w, z\rangle| \geqq 1$. Then $n$ can be chosen so that $z_{n}$ is within $1 / 2$ of $z$ on $K$, and so on $K^{\prime}$, so that $\left|\left\langle w, z_{n}\right\rangle\right| \geqq 1 / 2$. Thus $\|w\|>0$. Since every element of $W$ is a scalar multiple of an element in $K^{\prime}$, it follows that $\|w\|>0$ whenever $w \in W$ and $w \neq 0$.

We now show that on $K^{\prime}$ the norm topology coincides with the original topology from $V$. Suppose that $w_{a}$ is a net of elements of $K^{\prime}$ which converges to $w \in K^{\prime}$ in the original topology, and so in the weak topology. Given $\varepsilon>0$ choose $N$ large enough so that $\sum_{n>N} 2^{-n}<\varepsilon / 4$. Choose $a_{0}$ so that if $a>a_{0}$ then

$$
\left|\left\langle w-w_{a}, z_{n}\right\rangle\right| \leqq\left|z_{n}\right| \varepsilon / 2
$$

for $1 \leqq n \leqq N$. Then, if $a>a_{0}$,

$$
\left\|w-w_{a}\right\| \leqq \sum_{n=1}^{N} 2^{-n} \varepsilon / 2+2 \sum_{n>N} 2^{-n} \leqq \varepsilon .
$$


Thus on $K^{\prime}$ the norm topology is coarser than the original topology. But the original topology is compact and the norm topology is Hausdorff, so the two topologies must coincide.

COROLLARY 5.3. If $K$ is a compact subset of a locally convex space whose relative topology has a countable base, then the relative topology of the closed circled convex extension of $K$ has a countable base.

The following corollary is the particular version of Theorem 5.2 which is used to prove the Radon-Nikodym theorems of Dunford and Pettis [7] and Dieudonne [4] using our Main Theorem.

Corollary 5.4. Let $B$ be a separable Banach space with dual space $B^{*}$. Then a norm, \|\|$_{0}$, can be defined on $B^{*}$ such that on bounded (and so relatively $w^{*}$ compact) subsets of $B^{*}$ the \|\|$_{0}$-topology coincides with the $w^{*}$-topology.

Actually this corollary may be proved directly. If $\left\{b_{n}\right\}$ is a sequence of elements dense in the unit ball of $B$, then

$$
\left\|b^{*}\right\|_{0}=\sum_{n=1}^{\infty} 2^{-n}\left|\left\langle b_{n}, b^{*}\right\rangle\right| \quad \text { for } b^{*} \in B^{*}
$$

is such a norm.

The following corollary is the particular version of Theorem 5.2 which is used to prove the Radon-Nikodym theorem of Phillips [13] using our Main Theorem (but one must first prove the nontrivial fact that the hypotheses of Phillips' theorem imply separability, see [9, p. 426] or [3, p. 95, Exercise 24]).

Corollary 5.5. Let $B$ be a separable Banach space and let $K$ be a weakly compact subset of $B$. If $W$ is the linear manifold spanned by the closed circled convex extension $K^{\prime}$ of $K$, then a norm, \|\|$_{0}$, can be defined on $W$ such that on $K^{\prime}$ the \|\|$_{0}$-topology coincides with the weak topology.

Proof. By Theorem 3 of $[8$, p. 434] the weak topology of $K$ is metrizable, and so has a countable base.

We remark that Corollaries 5.4 and 5.5 in conjunction with our proof in $\S 4$ of the Kreinn-Milman theorem for norm compact sets yield a new proof of the KreinMilman theorem for $w^{*}$-compact subsets in the dual of a separable Banach space, and for weakly compact subsets of a separable Banach space.

Proof of Theorem 5.1. We can assume that $\mu$ is totally finite.

Let $W$ be the linear manifold spanned by the closed circled convex extension, $K$, of $A_{X}$. By Theorem 5.2 there is a norm, \|\| , on $W$ such that on $K$ the original topology coincides with the norm topology. Let $W_{0}$ be the completion of $W$ with respect to this norm, so that $W_{0}$ is a Banach space. Since $\bar{A}_{X}$ is compact in $W$ it is closed in $W_{0}$. Then $m$ can be viewed as a measure with values in $\mu(X) K \subseteq W_{0}$ clearly satisfying hypotheses (1) and (3) of the Main Theorem. Hypothesis (2) is 
also satisfied, for $\bar{A}_{X}(m)$ is bounded and so there exists a constant, $N$, such that

$$
\|m(E)\| \leqq N \mu(E) \text { for } E \in S .
$$

Then $|m|(X) \leqq N \mu(X)$, and since $\mu$ is totally finite, $|m|$ is also.

Then, applying the Main Theorem, there is a $W_{0}$-valued Bochner integrable function, $f$, such that $m=\mu_{f}$. Since according to Proposition $1.10 \mathrm{er}_{X} f \subseteq \bar{A}_{X}(m)$, we can, by Proposition 1.6, change $f$ on a null set so that $\operatorname{range}(f) \subseteq \bar{A}_{X} \subseteq V$. Furthermore, by Proposition 1.13, if, as before, we define

$$
f_{\pi}=\sum_{E \in \pi}(m(E) / \mu(E)) \chi_{E}
$$

for $\pi \in \Pi$, then $f_{\pi}$ converges to $f$ in mean. Then we can extract a subsequence, $f_{\pi_{n}}=f_{n}$ which converges to $f$ a.e. as well as in mean. In particular $\mu_{f_{n}}(E)$ converges in norm to $\mu_{f}(E)=m(E)$ for each $E \in S$.

We now show that for each $z \in V^{*}$ and each $E \in S$

$$
\langle m(E), z\rangle=\int_{E}\langle f(x), z\rangle d \mu(x) .
$$

If $\mu(E)=0$ the result is clear, so we may assume that $\mu(E)>0$. Since on $\bar{c}\left(A_{X}\right)$ the norm topology coincides with the original topology, $z$ is continuous on $\bar{c}\left(A_{X}\right)$, and so is bounded on $\bar{c}\left(A_{X}\right)$, say by $C$. It follows that $\langle f(\cdot), z\rangle$ is an integrable function bounded by $C$, as is $\left\langle f_{\pi}(\cdot), z\right\rangle$ for each $\pi$, since range $\left(f_{\pi}\right) \subseteq \bar{A}_{X}(m)$. In particular, $f$ is weakly integrable. Furthermore, since $f_{n}$ converges to $f$ a.e., it follows that

$$
\left\langle f_{n}(x), z\right\rangle \rightarrow\langle f(x), z\rangle \quad \text { a.e. } x .
$$

Then by the Lebesgue dominated convergence theorem

$$
\int_{E}\left\langle f_{n}(x), z\right\rangle d \mu(x) \rightarrow \int_{E}\langle f(x), z\rangle d \mu(x)
$$

for each $E \in S$. Now, since the $f_{n}$ are simple functions, it is easily verified that

$$
\int_{E}\left\langle f_{n}(x), z\right\rangle d \mu(x)=\left\langle\mu_{f_{n}}(E), z\right\rangle .
$$

Then, since $\mu_{f_{n}}(E) \rightarrow m(E)$ in norm, since $\mu_{f_{n}}(E) / \mu(E) \in \bar{c}\left(A_{X}\right)$ and since $z$ is continuous on $\bar{c}\left(A_{X}\right)$, it follows that

$$
\left\langle\mu_{f_{n}}(E), z\right\rangle \rightarrow\langle m(E), z\rangle .
$$

Thus

$$
\langle m(E), z\rangle=\int_{E}\langle f(x), z\rangle d \mu(x)
$$

for all $E \in S$ and $z \in V^{*}$, concluding the proof.

6. Examples. In this section we gather together a few examples which illustrate certain aspects of our results. In particular, they show that the hypotheses of the 
Main Theorem are independent. The first three examples are well known. In all of the examples $X=[0,1]$ and $\mu$ is Lebesgue measure on $X$.

Our first example shows that hypotheses (1) and (2) of the Main Theorem do not imply hypothesis (3) (or $\left.3^{\prime}\right)$ ).

EXAMPLE 6.1. Let $B=L^{1}(X, \mu)$, and define a $B$-valued measure, $m$, by $m(E)=\chi_{E}$ for each Lebesgue measurable set $E$. Then $m$ is $\mu$-continuous, in fact, $|m|=\mu$. But it is easily checked that $A_{E}(m)$ is not relatively norm compact for any $E$ of strictly positive measure, so that $m$ is not the indefinite integral of any Bochner integrable function.

Our next example shows that hypotheses (1) and (3) (or (3')) do not imply hypothesis (2).

EXAMPLE 6.2 [1, Example 7, p. 377]. Let $B$ be an infinite dimensional Hilbert space, and let $\left\{e_{n}\right\}_{n=1}^{\infty}$ be an orthonormal system in $B$. Define a $B$-valued measure, $m$, by

$$
m(E)=\sum_{n=1}^{\infty}\left(2^{n} / n\right) \mu\left(E \cap\left(2^{-n}, 2^{-n+1}\right]\right) e_{n} .
$$

Then $m$ is clearly $\mu$-continuous, and $A_{(t, 1)}(m)$ is a bounded subset of a finite dimensional subspace of $B$ for any $t>0$, so that $m$ satisfies hypotheses (3) and ( $\left.3^{\prime}\right)$. But $|m|([0,1])=\sum_{n=1}^{\infty} 1 / n=\infty$, so that hypothesis (2) is not satisfied. We remark that if $\chi_{n}$ denotes the characteristic function of the interval $\left(2^{-n}, 2^{-n+1}\right]$, then $m$ is the indefinite integral of the function

$$
f=\sum_{n=1}^{\infty}\left(2^{n} / n\right) \chi_{n} e_{n}
$$

which is Birkhoff integrable [1] but not Bochner integrable, since $\|f(\cdot)\|$ is not Lebesgue integrable.

Our next example shows that hypotheses (2) and (3) (or (3')) do not imply hypothesis (1).

EXAMPLE 6.3. Let $B$ be the real numbers, and let $m$ be the unit mass at the point 0 . Then hypothesis (2) is clearly satisfied, and $A_{(t, 1]}(m)=\{0\}$ for any $t>0$ so that hypotheses (3) and ( $\left.3^{\prime}\right)$ are satisfied, but hypothesis (1) is clearly not satisfied.

Our next example serves two purposes. First, it shows that to characterize the indefinite integrals of even bounded Bochner integrable functions the word "somewhere" in hypotheses (3) and ( $\left.3^{\prime}\right)$ can not be omitted. Secondly it shows that the hypotheses of Phillips' Radon-Nikodym theorem [14, p. 134, Theorem 5.5], while sufficient, are not necessary for a measure to be the indefinite integral of a Bochner integrable function. Specifically, Phillips' hypothesis that for any constant $N$ the set

$$
K_{N}=\{m(E) / \mu(E): E \in S \text { and }\|m(E) / \mu(E)\| \leqq N\}
$$

should be weakly relatively compact, is not satisfied by the following example for $N \geqq 1$. 
EXAMPLE 6.4. Let $B$ be any nonreflexive Banach space, e.g. $l^{1}$, so that the unit ball of $B$ is not weakly compact. By Eberlein's theorem [8, p. 430] the unit ball of $B$ is not weakly sequentially compact, so there exists a sequence $\left\{b_{n}\right\}$ of elements of the unit ball of $B$ which is not weakly relatively compact. For example, in $l^{1}$ let $b_{n}$ be the unit mass at the integer $n$. Define the function $f$ on $X$ by

$$
f=\sum_{n=1}^{\infty} x_{n} b_{n}
$$

where $\chi_{n}$ has the same meaning as in Example 6.2. Then $f$ is a bounded Bochner integrable function. But if $m=\mu_{f}$, then $A_{X}(m)$, which is also the same as the set $K_{N}$ for $N \geqq 1$, contains $\left\{b_{n}\right\}$, and so is not weakly relatively compact, much less norm relatively compact.

In our next example we construct a measure which satisfies hypotheses (1) and (2) of the Main Theorem and whose range is relatively compact, but which is not the indefinite integral of a Bochner integrable function on any subset of $X$ of strictly positive measure. In particular, this shows that in hypothesis $\left(3^{\prime}\right)$ some condition must be imposed on $K$, such as disjointness from 0 .

EXAMPLE 6.5. Let $B=l^{\infty}$. Let $\left\{f_{i}\right\}$ be a uniformly bounded sequence of realvalued functions on $X$ which converges to 0 in $L^{1}(X, \mu)$ and which has the property that if $E$ is any subset of $X$ then the restrictions of the $f_{i}$ to $E$ are not equicontinuous at any nonisolated point of $E$. For example let the $f_{i}$ be the characteristic functions of the intervals $[0,1 / 2],[1 / 2,1],[0,1 / 3],[1 / 3,2 / 3]$, etc. [10, p. 94, Exercise 6]. Define an $l^{\infty}$-valued measure, $m$, on $X$ by

$$
(m(E))_{i}=\int_{E} f_{i} d \mu .
$$

Then it is clear that $m$ is $\mu$-continuous. Also $|m| \leqq c \mu$ where $c$ is a uniform bound for the $f_{i}$, so $|m|$ is finite. Furthermore the range of $m$ is contained in the set

$$
\left\{a \in l^{\infty}:\left|a_{i}\right| \leqq\left\|f_{i}\right\|_{1}\right\}
$$

which is totally bounded and so compact, since $\left\|f_{i}\right\|_{1}$ converges to 0 .

Finally, we indicate why $m$ can not be the indefinite integral of any Bochner integrable function on any set $E$ of strictly positive measure. If $m$ were the indefinite integral of some Bochner integrable function, $g$, on $E$ then it is not difficult to verify that $g$ must equal $f$ a.e. on $E$, where $f$ is defined by $(f(x))_{i}=f_{i}(x)$. But $f$ is not measurable on $E$, for if it were, then by Lusin's theorem [10] there would be a compact subset, $C$, of $E$ of measure arbitrarily close to $\mu(E)$ such that the restriction of $f$ to $C$ is continuous, that is, the $f_{i}$ are equicontinuous on $C$. Since $C$ must have nonisolated points, this contradicts the way in which the $f_{i}$ were chosen.

It is natural to ask whether the analogue of hypothesis (3a) for direction is a necessary condition, that is, whether the indefinite integral of a Bochner integrable function locally almost has compact direction. Our next example shows that this need not be the case. 
EXAMPLe 6.6. Let $H$ and $\left\{e_{n}\right\}$ be as in Example 6.2. For each $n$ let $g_{n}$ be the function on $X$ defined by

$$
g_{n}(x)=(-1)^{k} \text { for } k / 2^{n} \leqq x<(k+1) / 2^{n}
$$

where $0 \leqq k<2^{n}$. Let

$$
f=\sum_{n=1}^{\infty}\left(1 / 8^{n}\right) g_{n} e_{n}
$$

Then it is not difficult to show that for any $E \subseteq X$ with $\mu(E)>1 / 2$, the range of $\mu_{f}$ restricted to $E$ does not have compact direction.

\section{REFERENCES}

1. G. Birkhoff, Integration of functions with values in a Banach space, Trans. Amer. Math. Soc. 38 (1935), 357-378.

2. S. Bochner, Integration von Funktionen deren Werte die Elemente eines Vectorraumes sind, Fund. Math. 20 (1933), 262-276.

3. N. Bourbaki, Intégration, Chapitre 6, Actualités. Sci. Indust. No. 1281, Hermann, Paris, 1959.

4. J. Dieudonné, Sur le théorème de Lebesgue-Nikodym. V, Canad. J. Math. 3 (1951), 129-139.

5. L. E. Dubins, Generalized random variables, Trans. Amer. Math. Soc. 84 (1957), 273-309.

6. N. Dunford, Integration in general analysis, Trans. Amer. Math. Soc. 37 (1935), 441-453.

7. N. Dunford and B. J. Pettis, Linear operations on summable functions, Trans. Amer. Math. Soc. 47 (1940), 323-392.

8. N. Dunford and J. T. Schwartz, Linear operators, Part I, Interscience, New York, 1958.

9. A. Grothendieck, Espaces vectoriels topologiques, Sociedade de Mathematica de São Paulo, São Paulo, 1958.

10. P. R. Halmos, Measure theory, Van Nostrand, New York, 1950.

11. J. L. Kelley, I. Namioka, et al., Linear topological spaces, Van Nostrand, New York, 1963.

12. M. Metivier, Limites projectives de mesures. Martingales. Applications, Ann. Mat. Pura Appl. 63 (1963), 225-352.

13. R. S. Phillips, On weakly compact subsets of a Banach space, Amer. J. Math. 65 (1943), 108-136.

14. M. M. Rao, Decomposition of vector measures, Proc. Nat. Acad. Sci. U.S.A. 51 (1964), 771-774.

15. C. E. Rickart, An abstract Radon-Nikodym theorem, Trans. Amer. Math. Soc. 56 (1944), $50-66$.

16. A. and C. Ionescu Tulcea, On the lifting property. I, J. Math. Anal. Appl. 3 (1961), 537-546.

17. - On the lifting property. II, Representation of linear operators on spaces $L_{E}^{r}$, $1 \leqq r<\infty$, J. Math. Mech. 11 (1962), 773-796.

University of CALIFornia,

Berkeley, California 